Check for updates

Cite this: RSC Adv., 2018, 8, 32304

Received 1st July 2018

Accepted 2nd September 2018

DOI: $10.1039 / c 8 r a 05615 c$

rsc.li/rsc-advances

\section{The enhanced osteogenesis and osteointegration of 3-DP PCL scaffolds via structural and functional optimization using collagen networks}

\author{
Jinbing Wang, $\mathbb{D} \dagger^{\mathrm{a}}$ Chucheng Lin, $\hat{\dagger}^{\mathrm{b}}$ Xin Gao, ${ }^{\mathrm{c}}$ Zhiwei Zheng, ${ }^{\mathrm{a}}$ Mimgming Lv, ${ }^{\mathrm{a}}$ \\ Jian Sun*a and Zhiyong Zhang*def
}

Optimal balance between biological activity and mechanical stability should be meticulously considered during scaffold design for bone tissue engineering applications. To fabricate an individualized construct with biomechanical and biological functionality for bone tissue regeneration, a polycaprolactonecollagen $(\mathrm{PCL}-\mathrm{COL}$ ) composite construct was developed through the combination of three-dimensional printing (3-DP) technology and biomimetic collagen matrix incorporation, with a 3-DP PCL framework maintaining the mechanical stability and a porous collagen matrix improving the biological activity. The results indicate that the compressive modulus of the composite constructs increased synergistically (over $40 \mathrm{MPa}$ ), providing sufficient mechanical support during new bone formation. On the other hand, the collagen matrix with a micro-porous architecture structurally increased scaffold areas and provided cellular adhesion sites, allowing for the functional construction of a favorable 3D microenvironment for BMSC adhesion, proliferation and extracellular matrix production. Moreover, critical-sized long bone defect (CSD) implantation demonstrated that the optimized composite constructs could promote bone tissue regeneration (5.5-fold) and bone-material osteointegration (4.7-fold), and decrease fibrosis encapsulation, compared to pristine PCL. The results indicate that these biomimetically ornamented $\mathrm{PCL}-\mathrm{COL}$ constructs exhibit favorable mechanical properties and biological functionality, demonstrating great potential as an effective bone graft substitute for bone defect treatment. Meanwhile, they can also harness the advantages of 3-DP technology and a collagen-based functionalized strategy, facilitating the creation of customized and functional PCL-COL constructs for clinical translation.

\section{Introduction}

Bone tissue has a certain degree of regenerative capacity, but a critical-sized bone defect (CSD) caused by congenital disorders, traumatic injury or bone tumors is always accompanied with an unfavorable prognosis unless bone grafts are implanted. ${ }^{1}$ Currently available graft materials for critical-sized bone defect treatment include autogenous bone grafts, allogeneic bone and biomaterial-based bone graft. Bone autografts are the current 'gold standard', nevertheless the use of autografts is often limited by graft supply, unsatisfactory shape, and donorsite morbidity. Allogenic bone grafts circumvent the drawbacks of autografts, but they are beset by limitations related to disease transmission, tissue condition at the time of transplant, and concerns over immunogenicity. ${ }^{2,3}$ Inspired by the fascinating idea of generating autologous-like bone tissue substitutes, a series of biomaterials have been investigated, mimicking the extracellular matrix (ECM) of bone, providing biomechanical functionality and bioactivity to regenerate bone defects. ${ }^{\mathbf{4}-6}$

Currently, the most frequently used biomaterials to construct bone graft substitutes include ceramics, and natural 
or synthetic polymers and composites., ${ }^{2,7,8}$ Ceramic materials are used as biomaterial-based bone grafts primarily due to their excellent osteoconductivity and biocompatibility. Of the many ceramics available, the calcium phosphate (CaP) group, consisting of hydroxyapatite (HA) and tri-calcium phosphate (TCP), is the most commonly used. ${ }^{3}$ Ceramics display some problems, such as low fatigue resistance and low degradation controllability. ${ }^{9}$ Natural polymers (e.g., collagen, gelatin and silk) are advantageous for their biodegradability and favorable bioactivity, but are hindered by their weak mechanical properties for load-bearing applications. ${ }^{10}$ Synthetic polymers (e.g., PCL and PLGA) display favorable mechanical functionality, good processability and controllable biodegradability, but are limited by their inherent poor bioactivity, leading to poor cellular attachment and fibrosis encapsulation. ${ }^{\mathbf{1 1}}$ Therefore, to fabricate clinically related products that are translatable from bench to bedside, optimal balance between mechanical functionality, biological performance and fabrication processability must be considered as a priority.

As a synthetic polymer, polycaprolactone (PCL) has been used in medical devices for several decades years due to its versatility and processability. ${ }^{12}$ Previously, Zein and his group have developed three dimensional printed (3-DP) polycaprolactone (PCL) scaffolds for bone tissue engineering. ${ }^{13,14}$ These 3-DP PCL scaffolds display favorable mechanical properties and controllable processability, facilitating the fabrication of customized anatomical shapes and controllable porous architectures, and have been extensively used as bone graft substitutes in preclinical and clinical investigations. ${ }^{3,12,15,16}$ However, PCL is limited by its suboptimal biological interactions with cells in vitro and fibrosis encapsulation under in vivo implantation. ${ }^{17}$ To circumvent these limitations, inorganic phase (e.g., HA and $\beta$-TCP) incorporation strategies have been introduced. ${ }^{\mathbf{1 1}, \mathbf{1 8 - 2 0}}$ However, aggregation and phase separation lead to inhomogeneous dispersions and the masking of ceramic particles by the polymer, resulting in the exposure of the ceramic on the scaffold surface and the hindering of cell attachment and proliferation due to the hydrophobic nature of PCL. ${ }^{11,21}$ Previously, Pati $^{16}$ has fabricated cell-laid ECM ornamented 3DP PCL/ $\beta$-TCP scaffolds, overcoming the above disadvantages and supporting osteoblastic differentiation and promoting greater bone formation. This cell-laid ECM ornamenting, mimicking the ECM of bone tissue, showed tailorable physical properties and favorable bioactivity, however, this celllaid method is sophisticated and time consuming.

To fabricate a bone tissue substitute graft, satisfying the combination of mechanical functionality with biological performance, we hypothesized that a composite strategy would hold great promise. Firstly, a macro-porous PCL construct was fabricated via 3-DP techniques, and then micro-porous collagen (COL) networks were included to mimic the ECM of bone tissue. However, the macro-porous PCL framework served as the skeleton, providing temporary mechanical support to withstand physiological loading and protect the defect space for osteogenic cell and bone tissue ingrowth. Meanwhile, the microporous collagen networks mimicking the ECM of bone served as structural filling material spatially. Furthermore, these collagen network ornamented PCL struts functioned by providing cell attachment and proliferation sites, encouraging osteoconductivity and enhancing osteointegration with the host tissue. ${ }^{22}$ This hybrid fabrication strategy, in an effort to maximize the benefits of PCL and collagen, taking advantage of the high versatility, favorable mechanical properties and customizable fabrication of PCL, combined with the bioactivity of collagen, would match function with structure to achieve bone defect regeneration.

The objective of the current study is the functionalization of a 3-DP PCL scaffold through collagen (COL) incorporation and functionalization, and then to carry out evaluation via in vitro cell seeding and in vivo orthotopic implantation in rabbit radial CSDs. These PCL-COL composite constructs can not only harness the manufacturing advantages of 3-DP technology, especially the capability for custom-made shapes and sizes according to medical images, but can also resemble the ECM composition of natural bone, with favorable mechanical and biological characteristics; they have the potential to be an effective bone graft substitute for clinical bone defect treatment.

\section{Materials and methods}

\subsection{PCL scaffold fabrication}

A HTS Rapid Prototyping System was used to fabricate the PCL scaffolds. Briefly, PCL pellets (MW: 60 000; Shenzhen Esun Industrial, China) were fed into the stainless steel nozzle at a temperature of $120{ }^{\circ} \mathrm{C}$ with the application of a pneumatic pressure of $670 \mathrm{kPa}$ with a feeding rate of $10.0 \mathrm{~mm} \mathrm{~min}^{-1}$. Disc shaped (diameter of $10.0 \mathrm{~mm}$, height of $3.0 \mathrm{~mm}$ ) and cube shaped (diameter of $4 \mathrm{~mm}$, length of $15 \mathrm{~mm}$ ) PCL scaffolds were fabricated with a line width of $500 \mu \mathrm{m}$, a pore size of $1000 \mu \mathrm{m}$, and a line height of $250 \mu \mathrm{m}$, in a $0 / 60 / 120^{\circ}$ lay-down pattern, according a previous study. ${ }^{13}$

\subsection{Alkaline surface activation and collagen incorporation}

For scaffold surface activation, PCL scaffolds were immersed in $5 \mathrm{M} \mathrm{NaOH}$ solution $\left(37^{\circ} \mathrm{C}\right)$ for $24 \mathrm{~h}$ at a shaking speed of $80 \mathrm{rpm}$, then were repeatedly washed with Milli-Q water until a pH of 7.4 was obtained. ${ }^{23}$ The alkaline surface activated PCL is hereafter referred to as A-PCL. To further optimize the A-PCL constructs structurally and functionally, A-PCL scaffolds were immersed into collagen solutions of different concentrations $\left(10,20\right.$ and $\left.30 \mathrm{mg} \mathrm{mL} \mathrm{m}^{-1}\right)$, subsequently placed under vacuum for $10 \mathrm{~min}$ to remove entrapped air bubbles, and then frozen at $-80{ }^{\circ} \mathrm{C}$ and freeze-dried at $-50{ }^{\circ} \mathrm{C}$. The freeze-dried porous PCL-collagen composites (designated as PC-10, PC-20 and PC30 , according to the different collagen concentrations) were subsequently cross-linked using $50 \mathrm{mM}$ EDC (Sigma-Aldrich, China) solution $\left(\mathrm{H}_{2} \mathrm{O}\right.$ : ethanol $\left.=5: 95\right)$ for $24 \mathrm{~h}$, thoroughly washed in distilled water followed by $5 \mathrm{wt} \%$ glycine solution, rinsed again with water, and finally freeze-dried and stored.

\subsection{Scaffold morphology and porosity}

To characterize the morphologies of the scaffolds, samples $(n=$ 3 ) were gold coated for 1 min and observed under field-emission 
scanning electron microscopy (SEM, Hitachi, S-4800) at $10 \mathrm{kV}$; also, the pore sizes of the scaffolds were measured using SEM. The porosities of the scaffolds were calculated according to the equation: ${ }^{24}$

$$
\text { Porosity }=1-\frac{\rho_{\mathrm{ap}}}{\rho_{\mathrm{m}}}=1-\left(\frac{\omega_{\mathrm{PCL}}}{\rho_{\mathrm{PCL}}}+\frac{\omega_{\text {collagen }}}{\rho_{\text {collagen }}}\right) / V
$$

where $\rho_{\mathrm{m}}$ is the density of the scaffold, and $\rho_{\mathrm{ap}}$ is the apparent density of the scaffold. The densities of PCL and collagen are $1.145\left(\rho_{\text {PCL }}\right)$, and $1.32 \mathrm{~g} \mathrm{~cm}^{-3}\left(\rho_{\text {collagen }}\right)$, respectively. The weight percent ratios of PCL $\left(\omega_{\mathrm{PCL}}\right)$ and collagen $\left(\omega_{\text {collagen }}\right)$ were measured using an electronic micro-balance before and after the inclusion of collagen.

\subsection{Hydrophilicity and water absorption ability}

The surface hydrophilicity properties of the scaffolds were measured via water contact angle (WCA) measurements (automatic contact angle meter, model SL200B, Solon, China). At room temperature, a sessile ultra-pure water droplet with a volume of $2 \mu \mathrm{L}$ was dropped onto the scaffold. The contact angles of the scaffolds $(n=3)$ after $180 \mathrm{~s}$ were averaged and resulted in a mean \pm standard deviation. Water absorption abilities were evaluated via weighing the scaffolds before and after soaking in distilled water for $2 \mathrm{~h}$. The increased percentage of weight after water absorption was use to evaluate the water absorption ability, which was calculated according to the equation: ${ }^{9}$

$$
P=\left(W_{2 \mathrm{~h}}-W_{0}\right) / W_{0} \times 100 \%
$$

where $W_{2 \mathrm{~h}}$ and $W_{0}$ are the weights of the wetted scaffold and dry scaffold, respectively.

\subsection{Mechanical properties}

The mechanical properties of COL, PCL, A-PCL, and PC-10, 20 and 30 ( $n=3$ /group) were measured using an Instron 5542 universal tester (Instron Corp., Norwood, MA) with a $500 \mathrm{~N}$ load cell. All scaffolds were approximately $10 \mathrm{~mm}$ in diameter and $3 \mathrm{~mm}$ in height. The dried scaffolds were compressed at a loading rate of $1 \mathrm{~mm} \mathrm{m^{-1 }}$ to a strain level of $80 \%$ at room temperature. The compressive modulus values were calculated from the stress-strain curves, from the slopes of the initial linear portions of the curves, according to a previous study. ${ }^{\mathbf{1 0}}$

\subsection{In vitro cellular cytotoxicity, proliferation, and viability evaluations}

Rabbit bone marrow stromal cells (r-BMSCs) were obtained from 28 day-old fetal rabbits. $5 \times 10^{4}$ r-BMSCs in $100 \mu \mathrm{L}$ of Dulbecco's modified Eagle's medium (DMEM; Gibco, USA) with low glucose containing $10 \%$ fetal bovine serum (FBS; Gibco, USA) and 1\% penicillin streptomycin (D10 medium) were seeded onto the scaffolds in 6-well plates. For the quantification of seeding efficiency, the scaffolds were transferred to new plates $4 \mathrm{~h}$ after cell seeding; the cells attached to the plates were digested and counted $(n=6)$. Seeding efficiency was quantitatively assessed as a percentage of the cell fraction in the scaffolds relative to the amount of cells loaded per scaffold. To evaluate the cytotoxicity of the scaffolds, leaching liquor was prepared through immersing the scaffolds $(n=6)$ into $200 \mu \mathrm{L}$ of D10 medium for $24 \mathrm{~h}$ in a 48 well plate. r-BMSCs were seeded on a 96-well plate at a density of $10^{3}$ cells per well. After $24 \mathrm{~h}$, the medium of the test group was replaced either with the leaching liquor $(100 \mu \mathrm{L})$ acquired from scaffolds or fresh D10 medium (blank). After 3 days, the cytotoxicity of the scaffolds was assessed using Cell Counting KIT-8 (CCK-8; Dojindo, Kumamoto, Japan) at $450 \mathrm{~nm}$. The cell number was correlated to the optical density (OD). Analysis of cell viability and morphology from the scaffolds was performed with LIVE/DEAD Cell Imaging Kit staining. Briefly, the cell-seeded scaffolds were washed with PBS three times, and incubated in $2 \mu \mathrm{M}$ calcein-AM (staining live cells) and $4 \mu \mathrm{M}$ propidium iodide (PI, staining dead cells) in PBS for $30 \mathrm{~min}$ at $37^{\circ} \mathrm{C}$, before being washed again with PBS. Samples were imaged using a confocal laser scanning microscope (Leica, Germany) 7 days after cell seeding.

\subsection{In vivo study of the restoration of rabbit radial critical- size bone defects}

18 male New Zealand white rabbits with an average weight of 2.5 $\pm 0.2 \mathrm{~kg}$ and an age of 5 months were randomly divided into 2 groups ( $n=9$ for each group). Group A were implanted with APCL scaffolds; and Group B were implanted with PC-20. The animals were anesthetized with an ear marginal vein injection of $1.5 \%$ sodium pentobarbital (30 $\mathrm{mg} \mathrm{\textrm {kg } ^ { - 1 }}$ ). Under sterile conditions, the mid-shaft of the left radial bone was exposed through a lateral longitudinal skin incision and a section of the diaphysis $(15 \mathrm{~mm})$ was cut off using a dental drill with $0.9 \%$ saline rinsing to create a critical-size bone defect. ${ }^{25}$ Then, the prepared scaffolds (A-PCL and PC-20) were implanted. Muscles, fasciae and skin were separately closed over the defect with the use of 4-0 sutures. At time points 4, 8 and 12 weeks post-surgery, animals were anesthetized and underwent X-ray examination. The sequential fluorochrome markers Alizarin Red S (30 mg $\mathrm{kg}^{-1}$; Sigma-Aldrich) and calcein (30 $\mathrm{mg} \mathrm{kg}^{-1}$; Sigma-Aldrich) were administered via intraperitoneal injection 4 and 8 weeks post-surgery. All animals were sacrificed through an overdose of pentobarbital at 12 weeks to study the defect sites. The experiments followed the Principles of Laboratory Animal Care by the National Society for Medical Research and the Guide for the Care and Use of Laboratory Animals, published by the US National Institutes of Health Animals, and all protocols were approved by the Animal Care and Use Committee of Shanghai Ninth People's Hospital.

\subsection{Micro-CT evaluation}

Samples were fixed in $10 \%$ formalin for $48 \mathrm{~h}$, and rinsed with PBS several times. The fixed samples were examined and imaged using micro-CT to determine newly formed bone in the defects. The scanning parameters were set at $45 \mathrm{kV}$ and $80 \mathrm{~mA}$, with an exposure time of $3000 \mathrm{~ms}$ and a resolution of $20 \mu \mathrm{m} .3 \mathrm{D}$ images of the specimens were reconstructed from the scans using the micro-CT system software package. 


\subsection{Histological observations}

After evaluation with micro-CT, three samples were decalcified in $10 \%$ EDTA at $\mathrm{pH} 7.4$ for 4 weeks at $37^{\circ} \mathrm{C}$ and subsequently embedded in paraffin. Sections near the central area of the implants were used for haematoxylin and eosin (H\&E) and Masson's trichrome staining and were visualized using an optical microscope. Six other samples were dehydrated with ethanol and finally embedded in polymethylmethacrylate (PMMA). The embedded specimens were sectioned into $150 \mu \mathrm{m}$ thick sections using a Leica SP1600 saw microtome (Leica, Hamburg, Germany) along the long axis of the radius in the central region. These sections were subsequently ground and polished to a final thickness of about $40 \mu \mathrm{m}$ for fluorescence labeling observation under a confocal laser scanning microscope. The excitation/emission wavelengths of the chelating fluorochromes of 543/617 $\mathrm{nm}$ and $488 / 517 \mathrm{~nm}$ were used for Alizarin Red S (red), and calcein (green), respectively. ${ }^{26}$ Finally, undecalcified sections were stained using van Gieson's picrofuchsin.

\subsection{Histomorphometric analysis of bone tissue ingrowth and fibrous tissue encapsulation}

Image Pro Plus (Media Cybernetics, Silver Springs, MD, USA) was used to calculate the areas of new bone, specifically, the total area $(T)$, scaffold area $(S)$, new bone area $(B)$, and interspace area $(I)$. Total areas were defined according to the scaffold width and height in each section $(\Phi=4 \mathrm{~mm} \times 15 \mathrm{~mm})$, which includes the scaffold area, new bone area and interspace area. Scaffold areas were the areas covered by the PCL rods. Bone areas showed areas covered by new bone, stained in magenta. Interspace areas were defined as hollow spaces among the new bone and scaffold rods; hence this is where the fibrous tissue grows, according to a previous study. ${ }^{27}$

Percentage of new bone $=$ new bone area/total area $(B / T)$

$$
B / T=\frac{\text { new bone area }}{\text { total area }}
$$

Percentage of scaffold area $=$ scaffold area/total area $(S / T)$

$$
S / T=\frac{\text { scaffold area }}{\text { total area }}
$$

Percentage of interspace area $=$ interspace area/total area $(I / T)$

$$
I / T=\frac{\text { interspace area }}{\text { total area }}
$$

The bone mineral deposition rate was calculated from the distance between the two fluorescence (green and red) labels $\left(\mu \mathrm{m} \mathrm{day}{ }^{-1}\right)$.

\subsection{Histomorphometric analysis of osteointegration}

Additionally, the new bone and scaffold perimeters were measured using Image Pro Plus. The osteointegration capacity of the scaffold was evaluated by calculating the length of the bone-material contact surface/scaffold perimeter (CS/SP) ratio, where CS was calculated according to the following equation: ${ }^{5,27}$

$$
\mathrm{CS}=\frac{\mathrm{SP}+\mathrm{BP}-\mathrm{BSP}}{2}
$$

where SP: scaffold perimeter; BP: bone perimeter; and BSP: bone and scaffold perimeter.

\subsection{Statistical analysis}

All data presented are expressed as means \pm SD. Statistical analyses consisted of single-factor analyses of variance (ANOVA), or paired $t$-tests. The significance level was set at $p<$ 0.05 .

\section{Results}

\subsection{Characterization of the scaffolds}

The PCL constructs fabricated via 3-DP techniques display honey-comb like patterns and completely interconnected macroporous architectures, with a vertical pore size of $\sim 1000 \mu \mathrm{m}$ (top views, Fig. 1a and b), and a horizontal pore size of $340 \mu \mathrm{m}$ $\times 550 \mu \mathrm{m}$ (lower view, Fig. 1c). The A-PCL scaffold (after $\mathrm{NaOH}$ treatment) shows a similar morphology and architecture (Fig. 1e-g) to pristine PCL under macroscopic and lower SEM magnification observations. Under higher magnification, however, the A-PCL constructs display increased surface roughness compared to untreated PCL (Fig. 1d and h). The surface topography change is because hydroxide anions from $\mathrm{NaOH}$ solution hydrolyze the ester bonds of PCL, thereby exposing carboxylic $(-\mathrm{COOH})$ acid and hydroxyl $(-\mathrm{OH})$ groups through breaking the polymer chains. ${ }^{28}$ The results indicate that alkaline surface treatment is an effective method to modify the $3 \mathrm{D}$ porous polymer scaffold through surface chemical and topographical changes, without influencing the scaffold's porous architecture.

When the alkaline modified A-PCL was further functionalized with collagen incorporation, the macroporous A-PCL was completely filled with micro-collagen networks (Fig. 2A(a, e and i)). According to previous studies, the pore size of collagen can be tailored through controlling the final freezing temperature in the chamber of the freeze dryer, the pore size distribution is determined by the cooling rate, and a greater concentration of collagen gel leads to the formation of smaller pore sizes. ${ }^{5,29}$ In our study, SEM observations indicated microporous collagen networks homogeneously cross-embedded into the voids of the A-PCL struts both at the surface (Fig. 2A(b, f and j)) and in central areas (Fig. 2A(c, g and k)). The collagen matrix displays pore sizes of $132 \pm 17.6$ (PC-10), $126 \pm 18.3$ (PC-20) and $121 \pm$ $13.6 \mu \mathrm{m}$ (PC-30), and the pore size change is not significant. On the other hand, the A-PCL struts were coated with layers of micro-collagen film (Fig. $2 \mathrm{~A}(\mathrm{~d}, \mathrm{~h}$ and $\mathrm{l})$ ), with thicknesses of 2.1 $\pm 0.27,2.51 \pm 0.58$ and $3.50 \pm 0.29 \mu \mathrm{m}$ in the PC-10, 20 and 30 constructs, respectively. This indicated that collagen incorporation could structurally maximize the scaffold area, providing favourable cell attachment sites and proliferation spaces. Besides, pore size analysis (Fig. 2B) shows that collagen incorporation significantly decreases the pore size (macropore size: 

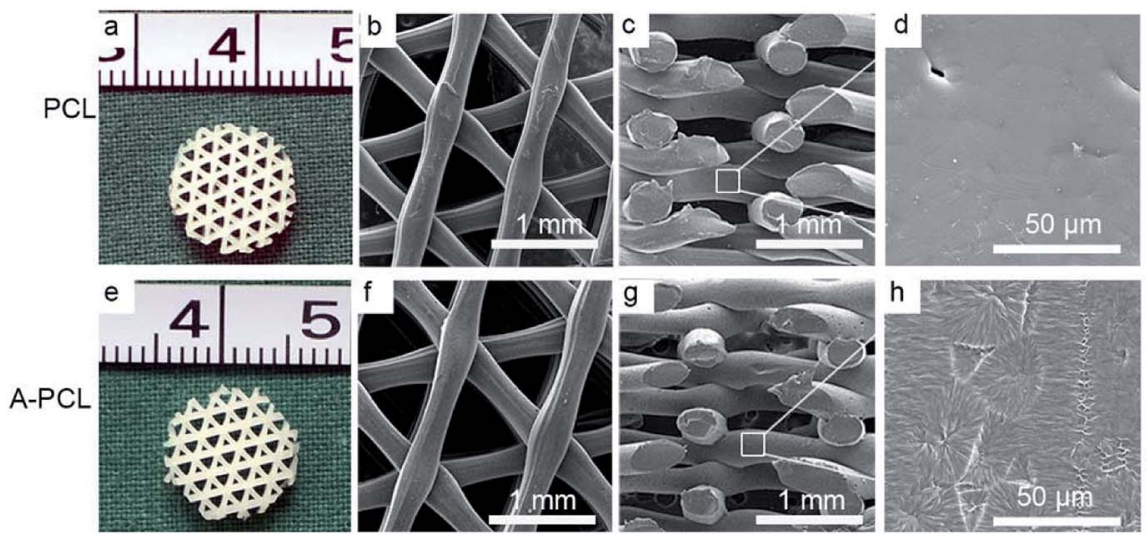

Fig. 1 Optical and SEM images of constructs pre \& post-alkaline ( $\mathrm{NaOH}$ ) treatment. Macroscopic (a and e) and low magnification SEM (b, c, fand g) images of PCL and A-PCL show similar surface appearances and porous architectures. (d and h) High magnification SEM images reveal that alkaline treatment significantly modified the surface of $A-P C L$, with its nano-topographical rough appearance compared to pristine PCL with its smooth surface.

(A)
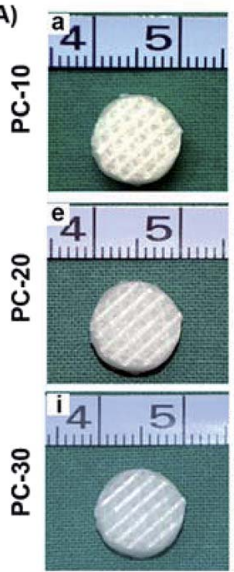

(B)

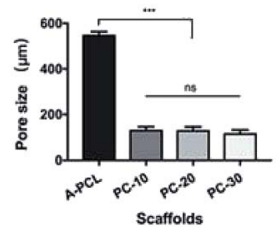

(C)
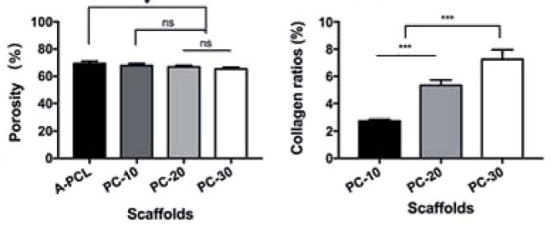

Fig. 2 Characterization of the $\mathrm{PCL}-\mathrm{COL}$ composite constructs. (A) Optical and SEM images of the constructs. Macroscopic (a, e and i) and SEM images (top view: $b, f$ and $j$; lateral view: $c, g$ and $k$ ) show that the composite constructs were filled with the homogeneous collagen matrix cross-embedded among the PCL struts, with layers (several micrometers thick) of collagen film coating the PCL struts ( $d, h$ and $l$ ). (B) An analysis of the pore size and (C) porosity of the composite constructs post-alkaline treatment and collagen functionalization. (D) An analysis of collagen ratios (W\%) after collagen incorporation at different concentrations. (*: $p<0.05$; ***: $p<0.001$; ns: non-significance; PC-10, PC-20 and PC-30 represent A-PCL constructs incorporated with different concentrations of collagen: 10,20 and $30 \mathrm{mg}$ $\mathrm{mL}^{-1}$ ).

$1000 \times 340 \times 550 \mu \mathrm{m})$ of A-PCL to an optimal one (a collagen matrix with a micropore size of $100-200 \mu \mathrm{m}$ ) especially favouring bone tissue ingrowth, according to a previous study. ${ }^{2}$ The collagen ratio analysis (Fig. 2D) indicates a significantly $(p<$ 0.001) increased collagen mass after the incorporation of different concentrations of collagen gel $\left(10,20\right.$ and $30 \mathrm{mg} \mathrm{mL}^{-1}$ collagen gel), with collagen ratios of $2.7 \pm 0.1 \%, 5.4 \pm 0.4 \%$ and $7.2 \pm 0.7 \%$, respectively. However, the increased collagen matrix didn't influence the porosity dramatically. The A-PCL constructs display a porosity of $69 \pm 1.8 \%$, which slightly $(p>0.05)$ decreased to $67.8 \pm 1.5 \%$ (PC-10), $66.8 \pm 1.1 \%$ (PC-20) and 65.4 \pm 1.2 (PC-30) (Fig. 2C).

The hydrophilic properties of the constructs were evaluated via water contact angle (WCA) analysis. The alkaline modified APCL displayed significantly greater hydrophilic properties (with a WCA of $23 \pm 3.2^{\circ}$ at $180 \mathrm{~s}$ ) compared to pristine PCL (with a WCA of $78 \pm 4.1^{\circ}$ at $180 \mathrm{~s}$; Fig. $3 \mathrm{~A}$ and B). This improvement in hydrophilic properties is ascribed to the chemical and topographical modification with $\mathrm{NaOH}$, because of the rough morphology (Fig. 1h) and exposure of hydrophilic carboxyl $(-\mathrm{COOH})$ and hydroxyl $(-\mathrm{OH})$ groups after alkaline treatment. ${ }^{30}$ The WCAs of PC-10, PC-20 and PC-30 were $0^{\circ}$ (Fig. 3A and B), indicating that the hydrophilicity of the scaffolds is further improved after collagen incorporation ( $p<0.001$; Fig. 3A and B). Compared to PCL $(43.22 \%)$, A-PCL $(65.78 \%)$ showed greater (1.52-fold) water uptake ability, which further improved after collagen incorporation; PC-10, PC-20 and PC-30 display water uptake ratios of $80.71 \%, 86.15 \%$ and $89.56 \%$, respectively (Fig. 3C). The water uptake ability of the scaffolds is defined as the ability to maintain water and for water permeation, which influence the transfer of nutrients and cell proliferation. ${ }^{9}$ Therefore, an improvement in the hydrophilic properties and water uptake capacity through collagen functionalization could promote the bioactivity and osteoconductivity of the scaffolds in vitro and in vivo.

As load-bearing tissue, the mechanical stability of scaffolds is very important for bone tissue regeneration. To evaluate the mechanical properties of the constructs, compression testing was carried out. The results indicate that PCL has a compressive modulus of $39.06 \pm 5.69 \mathrm{MPa}$, which slightly $(p>0.05)$ 
(C) Water uptake ability

(A)

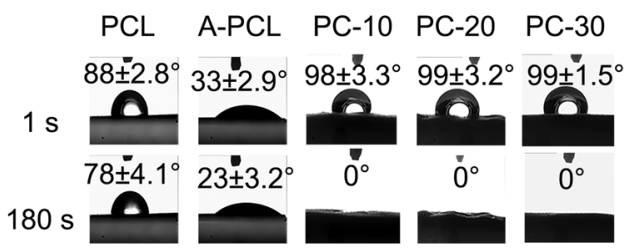

(B)

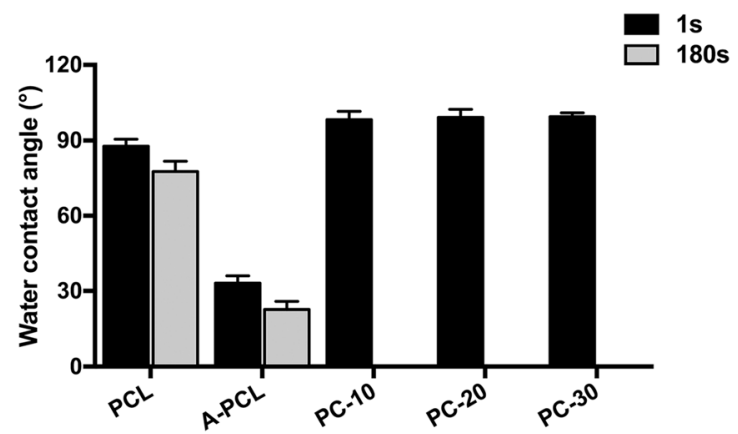

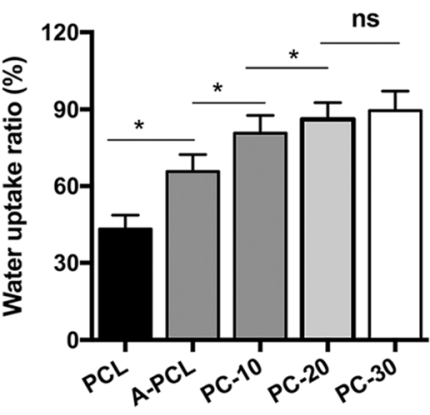

(D) Mechanical property

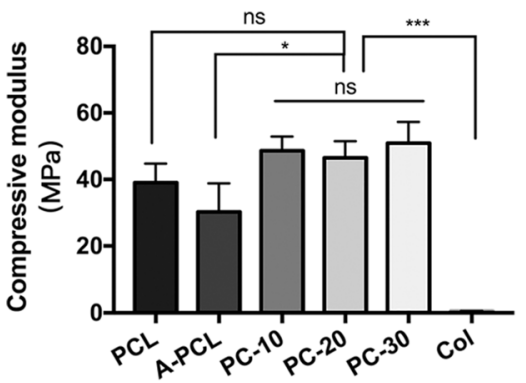

Fig. 3 An evaluation of the hydrophilic and mechanical properties of the constructs. (A) Water contact angle (WCA) measurements and (B) an analysis of the WCA values of the constructs. (C) Water uptake ability and (D) an analysis of the compressive modulus values of the constructs (*: $p$ $<0.05$; **: $p<0.001$; ns: non-significance).

decreased to $30.23 \pm 8.57 \mathrm{MPa}$ after alkaline treatment (A-PCL). Upon collagen incorporation and functionalization, the compressive modulus values of the PC-10, 20 and 30 constructs slightly increase to $48.61 \pm 4.30,46.48 \pm 5.06$ and $50.89 \pm$ 6.43 MPa, respectively. The results indicate that the compressive modulus values of the composites increase synergistically (up to 200-fold) compared with collagen sponge scaffolds alone (with a compressive modulus of $0.23 \pm 0.08 \mathrm{MPa}$ ). The results show that the composite constructs, with mechanical properties falling into the stiffness range of native spongy bone, could provide mechanical protection during new bone formation ${ }^{17}$ (Fig. 3D).

\subsection{In vitro cellular evaluation}

The cytotoxicity of the scaffolds was evaluated via CCK-8 assays; the results indicate that all scaffolds display favourable biocompatibility without cytotoxicity after cell culturing with extracted fluid for $72 \mathrm{~h}$ (Fig. 4A). From the cell adhesion ability assays, the results indicate that alkaline modification (A-PCL) significantly $(p<0.05)$ improves cell encapsulation capabilities compared to PCL (9.12 vs. 36.99\%), with further collagen functionalization resulting in significantly higher cellular adhesion capabilities (36.99 vs. 82.04\%) compared to A-PCL (Fig. 4B). However, there is no difference between the PC-10, 20 and 30 composite constructs with regards to cell encapsulation capabilities. The cell proliferation was significantly greater on the collagen incorporated scaffolds (Fig. 4C), possibly because the collagen matrix networks provide more cell adhesion sites and spaces for cell recognition and proliferation. The morphology, viability, and cell distribution of r-BMSCs cultured on the constructs were observed via contrast and confocal laser microscopy 7 days after cell seeding. Many more live cells were observed distributed on the PC-10, 20 and 30 composite constructs compared to the A-PCL scaffold (Fig. 4D), but there is no significant difference between the PC-10, 20 and 30 scaffolds. As the in vitro cellular studies demonstrate, there is no difference between the PC-10, 20 and 30 composite constructs. Finally, we selected PC-20 as a representative for the following in vivo observations.

\subsection{X-ray and micro-CT evaluations of critical-sized bone defect treatment}

X-ray images were used to evaluate bone tissue regeneration at different time points $(4,8$, and 12 weeks) after surgery. The results demonstrate that the bone defects retained radiolucency until 12 weeks in the A-PCL group, indicating no bone regeneration, whereas the PC-20 implantation achieved partial bridging of the defect at 4 weeks and presented full defect regeneration at 12 weeks (Fig. 5A). Additionally, the micro-CT results further confirmed that although the A-PCL scaffold presented a few bone calluses surrounding the A-PCL scaffold at the edges of the defects in the reconstruction images, there is no new bone tissue growth into the center of the scaffold in the longitudinal and cross section 
(A)

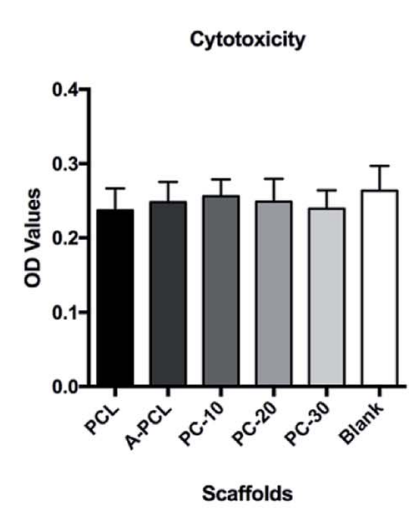

(D)

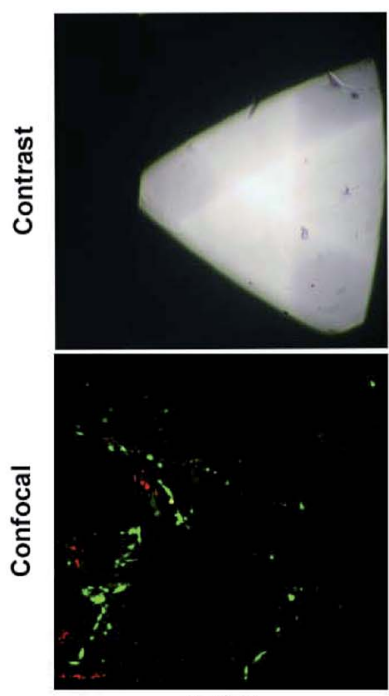

A-PCL
(B)

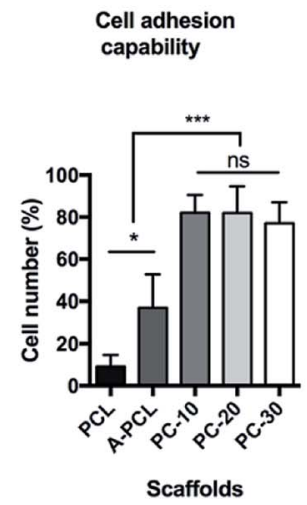

Scaffolds
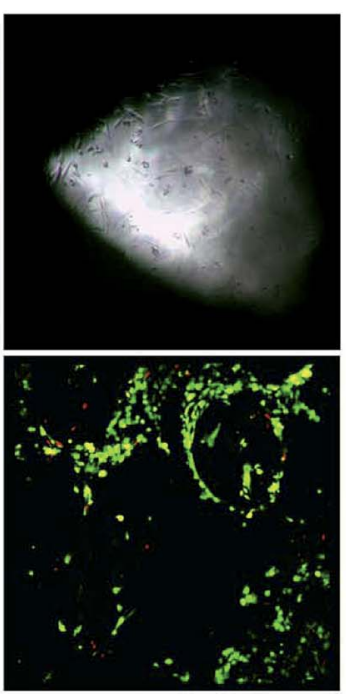

PC-10
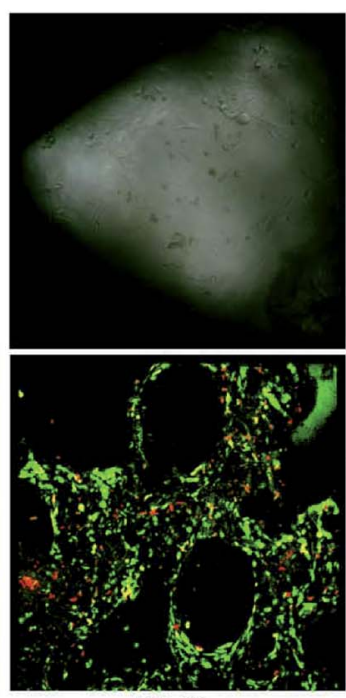

PC-20
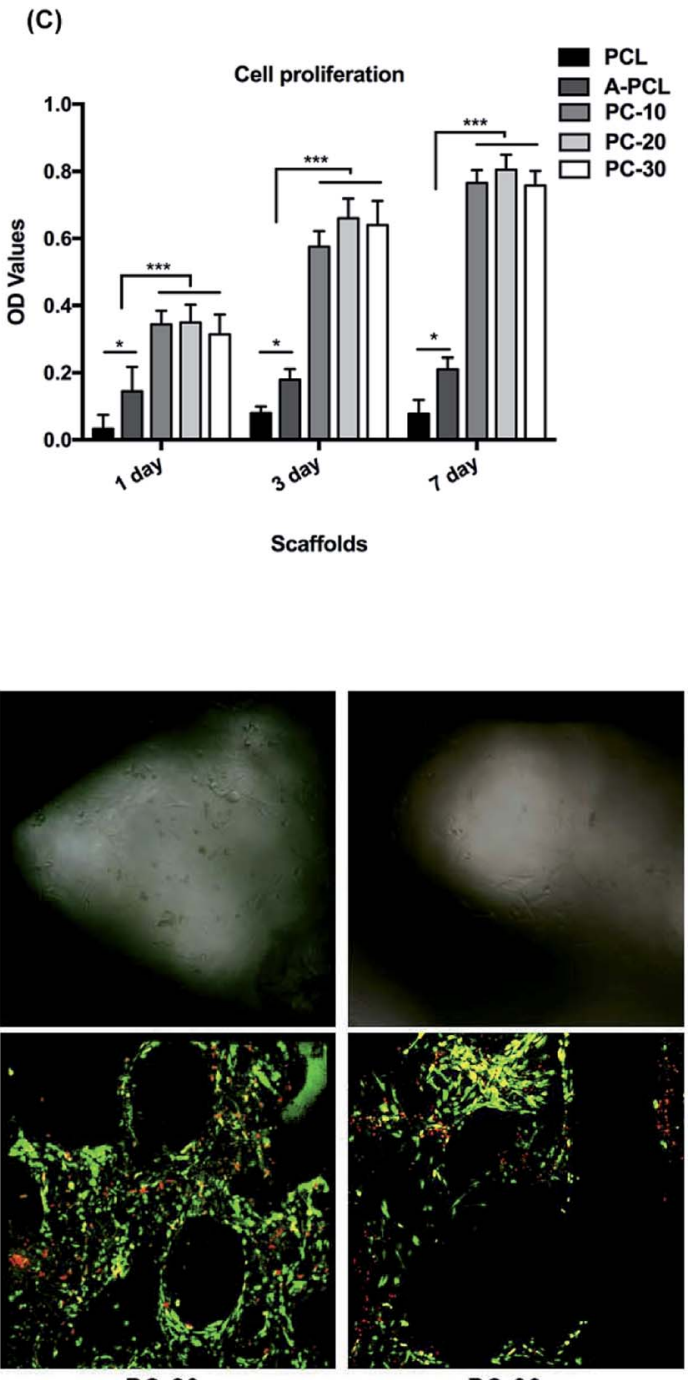

PC-30

Fig. 4 In vitro cellular evaluation of the biocompatibility and bioactivity of the constructs. (A) Cytotoxicity evaluation of the scaffolds with extracted liquor. (B) Cell adhesion capability assays. (C) Cell proliferation assays using CCK- 8 kits. (D) The cell morphology and distribution on the scaffolds were observed via contrast and confocal microscopy imaging 7 days after cell seeding (green color: live cells, red color: dead cells; *: $p$ $<0.05 ; * * *: p<0.001 ;$ ns: non-significance).

images (Fig. 5B). In the PC-20 scaffold group, the results indicate that successful defect bridging occurred, with partial circumferential cortical regeneration, and new bone tissue was observed surrounding and integrating with the scaffolds through the defect in the reconstruction images; there is an amount of new bone tissue growth into the center of the scaffolds, with functional osteointegration, visible in the longitudinal and cross section images (Fig. 5B). Moreover, quantitative measurements of the micro-CT data were utilized to analyse the bone ingrowth into defect areas; the results indicate that the PC-20 scaffold presents a significantly greater new bone volume, by 4.3 fold (135 \pm 25.6 vs. 31 $\pm 10.4 \mathrm{~mm}^{3}$ ), with a 2.77 fold increase in the $\mathrm{BV} / \mathrm{TV}$ ratio $(45.13 \pm 5.47$ vs. $16.24 \pm 3.36)$ compared to the A-PCL group (Fig. 5C and D).

\subsection{Histomorphometric analysis of bone tissue ingrowth and osteointegration}

Histological analysis was further conducted to evaluate the osteoinductive and osteointegration capabilities of the composite scaffolds. HE staining was used to investigate bone regeneration in the boundary areas between the scaffolds and host bones (Fig. 6A). The results indicate that PC-20 construct implantation resulted in much more new bone tissue (NB) regeneration; the porous collagen had been fully absorbed, and the spaces among the PCL struts were filled with newly formed bone ingrowth, presenting functional integration between the PCL scaffolds and HB (host bone) directly (Fig. 6A(a and b), black arrows). In contrast, A-PCL scaffold implantation resulted in no new bone tissue regeneration in the defect areas; the boundary areas (among the scaffolds and host bone) were occupied by fibrous connective tissue, which encapsulated the 
(A) X-ray images

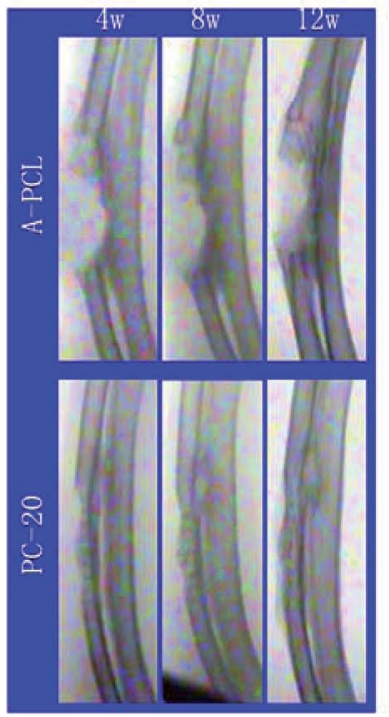

(B) Micro CT images

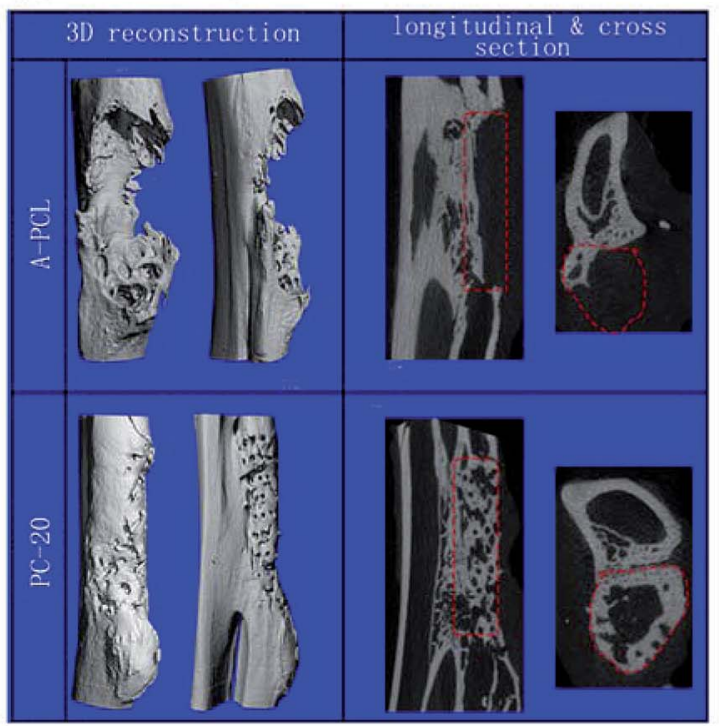

(C)

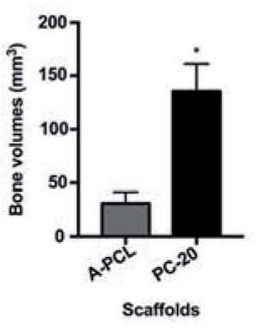

(D)

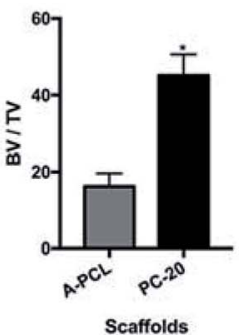

Fig. 5 A radiological evaluation of the constructs for rabbit radial critical-sized long bone defect treatment. (A) X-ray examinations at different time points (4, 8 and 12 weeks) post-implantation. (B) Micro-CT images at 12 weeks (column 1: lateral and front views of 3D reconstructed images; column 2: longitudinal section views parallel to the long axis of the bone and cross-sectional views of the center of the defect). (C) Quantitative micro-CT analysis of new bone formation. PC-20 scaffolds have successfully repaired critical-sized radial bone defects, with complete defect bridging, and achieved significantly higher new bone regeneration compared to A-PCL. (D) Bone volume versus total volume for the scaffold (BV/TV) from micro-CT analysis. (*: $p<0.05$; the red dotted lines indicate the contours of the bone defect in longitudinal and cross section images).

struts of the A-PCL constructs. The host bone-material interface was separated by a layer of fibrous tissues with a thickness of $>100 \mu \mathrm{m}$ (Fig. 6A(c and d)), hindering new bone tissue growth in the defect areas. Fibrous tissue encapsulation prevents interaction with the surrounding microenvironment, compromising bone tissue ingrowth and material-host bone functional integration. ${ }^{31}$ Masson's trichrome staining was further used to analyse bone regeneration in the central areas of the defects
(A) H\&E
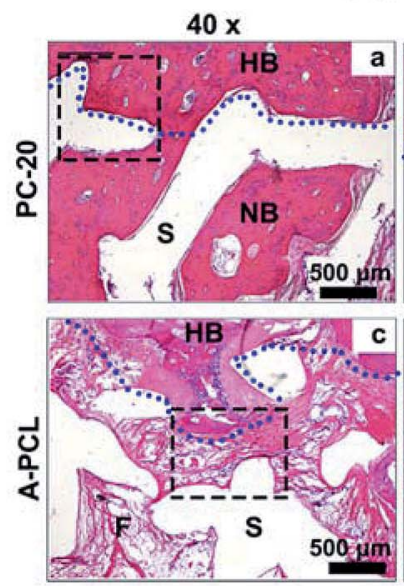

$100 x$
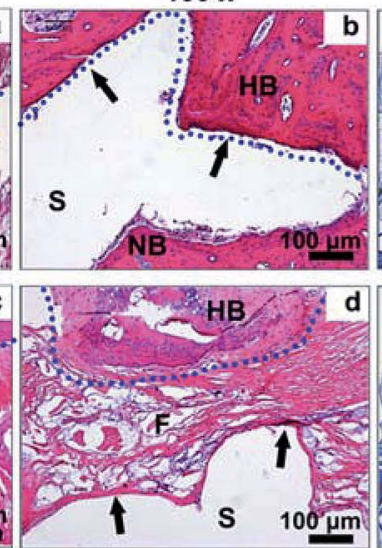

(B) Masson's Trichrome

$40 x$
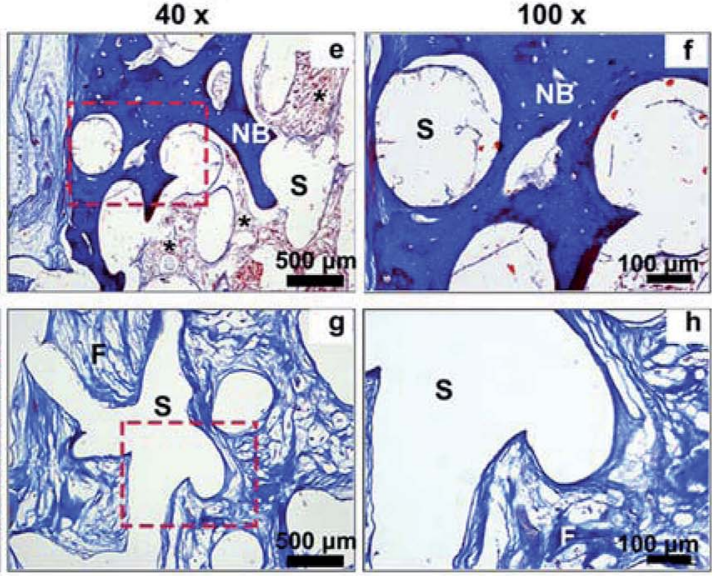

s.

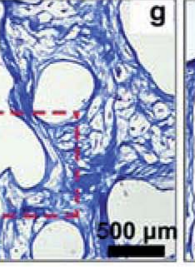

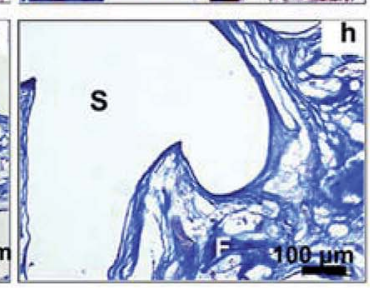

Fig. 6 In vivo histological evaluation. (A) H\&E staining analysis of the interface areas (blue dotted lines) between the host bone (HB) and defects areas. PC-20 implantation obtains better new bone tissue (NB) growth in the pores of the constructs and presents intimate integration with the A-PCL struts directly ( $a$ and b, black arrows). However, in the A-PCL group, the interface was occupied by fibrous connective tissue encapsulating the struts of the A-PCL constructs, where the host bone-material interface was separated by fibrous tissue (c and d, black arrows). (B) Masson's trichrome staining analysis of bone regeneration in the central areas of the defects. It is demonstrated that implantation of PC-20 constructs achieved complete defect bridging, with much more bone tissue formation and better osteointegration between the material and bone tissue in the center areas (e and f); furthermore, a bone-marrow-like structure (e, black stars) was found, indicating the generation of mature bone structure. In the A-PCL group, the defects were completely filled with loose fibrous tissue without any bone regeneration ( $g$ and $h$ ). (S: scaffolds; NB: newly formed bone; FB: fibrosis; HB: host bone). 
(Fig. 6B). It is demonstrated the implantation of PC-20 constructs achieved complete defect bridging and more new bone tissue formation. Also, in the central areas of the defects, the PCL struts were surrounded and integrated with newly formed bone tissue (Fig. 6B(e and f), blue); furthermore, a bonemarrow-like structure (Fig. 6B(e), black stars) was found as well, indicating the generation of a mature bone structure. In the APCL group, the defects were completely filled with loose fibrous tissue (Fig. 6B(g and h)).

To further evaluate new bone regeneration, un-decalcified samples were stained with Van Gieson's picrofuchsin staining for histomorphometric and quantitative analysis (Fig. 7). Newly formed bone tissue was stained in red with a woven, trabecular appearance, while the fibrous tissue filling the interspace areas was stained blue. The results indicate that PC20 composite construct implantation promotes better bone tissue regeneration and less fibrosis encapsulation, compared to A-PCL implantation, with fewer red areas but larger blue areas in the defects (Fig. 7A). Quantitative analysis further confirms that PC-20 implantation presents much more bone regeneration with significantly higher $B / T$ (5.5-fold, $59.99 \pm$ $8.95 \%$ vs. $10.87 \pm 6.13 \%)$ and lower $I / T(12.83 \pm 7.40$ vs. 52.66 $\pm 5.66 \%$ ) values, compared to A-PCL implantation, but there is no difference between the $S / T$ values, indicating that PCL degradation rates were similar in the PC-20 and A-PCL groups. In sequential fluorescent labelling observation images (Fig. 7C), red (Alizarin) and green (calcein) fluorescent labelling signals were detected throughout the entire defect area for the PC-20 group, suggesting active new bone deposition at 4 and 8 weeks, whereas fluorescent labelling was observed merely at the margins of the defect area in the A-PCL group. The distance between the white dotted line (representing the centre of the newly formed bone tissue at 4 weeks) and green line was measured to evaluate the mineral deposition rate. The PC-20 group showed a higher new bone deposition rate, 1.8 times faster $(6.15 \pm 1.03 v s .3 .29 \pm 0.93 \mu \mathrm{m}$ per day $)$ than that of the A-PCL group between 4 and 8 weeks.
(A)
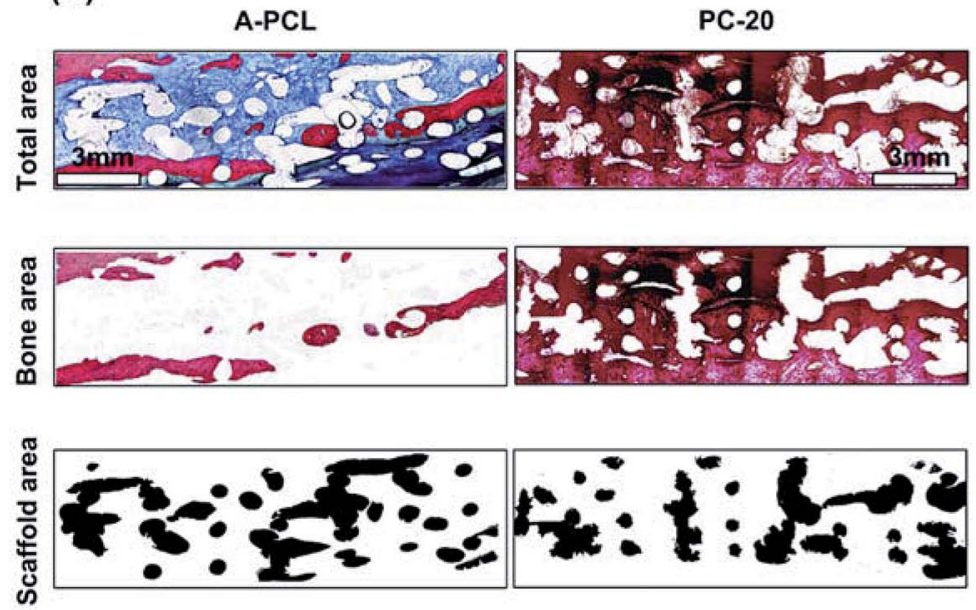

(C)
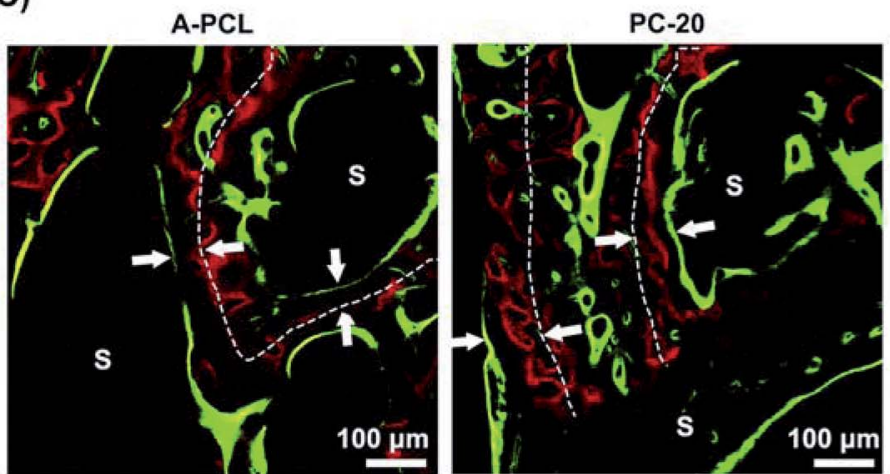

(B)

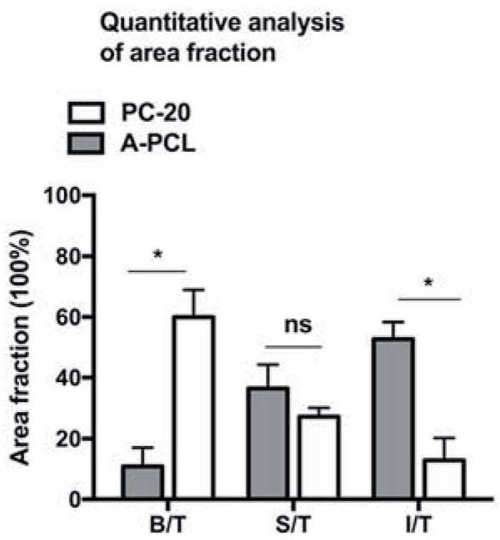

(D)

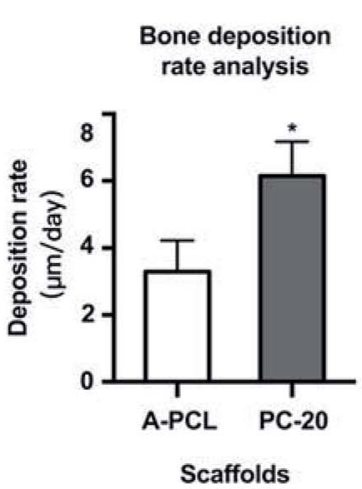

Fig. 7 Histomorphometric analysis of bone tissue regeneration. (A) Van Gieson's picrofuchsin staining observations indicate that the PC-20 composite constructs result in much more bone regeneration, with less fibrosis encapsulation. (B) A quantitative analysis indicates that PC-20 implantation results in significantly higher $B / T$ ( 5.5 -fold, $59.99 \pm 8.95 \%$ vs. $10.87 \pm 6.13 \%$ ) and lower $1 / T(12.83 \pm 7.40$ vs. $52.66 \pm 5.66 \%)$ values, compared to A-PCL implantation, but there is no difference regarding the S/T value. (C) Sequential fluorescent labelling (Alizarin Red S: red; calcein: green) observations indicate new bone deposition at 4 and 8 weeks. (D) The distances between the red lines (white dotted lines) and green lines were measured to evaluate the mineral deposition rate (white arrows). The white dots represent the central lines of bone tissue deposited at 4 weeks. (B/T: new bone area/total area; S/T: scaffold area/total area; I/T: interspace area/total area; S: scaffolds; *: $p<0.05 ;$ ns: not significant). 
Furthermore, the osteointegration of the scaffolds was evaluated quantitatively through calculating the bone and scaffold contact surface versus the scaffold perimeter ratio (CS/SP), according to our previous study. ${ }^{5,27}$ It is demonstrated that the PC-20 composited scaffold presented a 4.7-fold higher degree of bone-material osteointegration than the A-PCL scaffold, with a CS/SP value of $67.46 \pm 15.09 \%$ for the PC-20 group compared to $14.27 \pm 7.13 \%$ for the A-PCL group ( $p<0.05$, Fig. $8 \mathrm{E}$ ). The results reveal that $\mathrm{PC}-20$ implantation results in better osteointegration.

\section{Discussion}

To fabricate bone graft substitutes with optimal mechanical properties and custom-tailored architectures, rapid prototyping (RP) technology has emerged as a promising technique. ${ }^{17,32,33}$ This allows for fully computerized design and the precise fabrication of implants that are highly reproducible and fully customizable with shape, size, and desired internal microstructure set according to medical images, such as CT or MRI images. Among existing RP technologies, FDM based 3-DP technology demonstrates advantages for clinical application; in particular, it allows for a solvent-free fabrication process for thermoplastic polymers, such as PCL, minimizing concerns about the potential adverse effects of residual solvents after in vivo implantation. ${ }^{34}$ Previously, 3-DP fabricated PCL-TCP scaffolds with a unique honey-comb interconnected structure possessing similar mechanical properties to cancellous bone have been utilized as bone substitute grafts, demonstrating the potential to promote bone healing for both preclinical and clinical applications. ${ }^{3,13,14,27}$

In the present study, a 3-DP fabricated mechanically stabilized macro-porous PCL construct served as the framework to withstand surrounding tissue contraction and physiological loading. The native PCL constructs displayed a compressive modulus of $39.06 \pm 5.69 \mathrm{MPa}$, which slightly decreased after
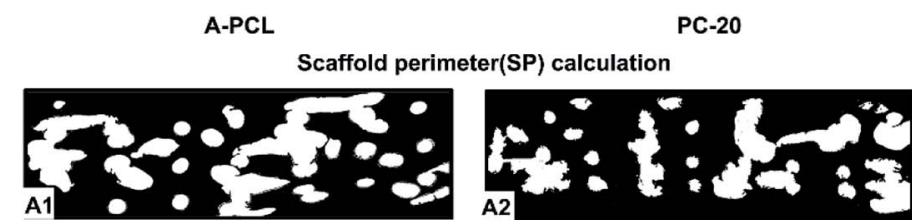

Schematic illustration

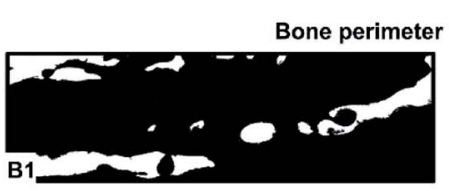

(BP) calculation
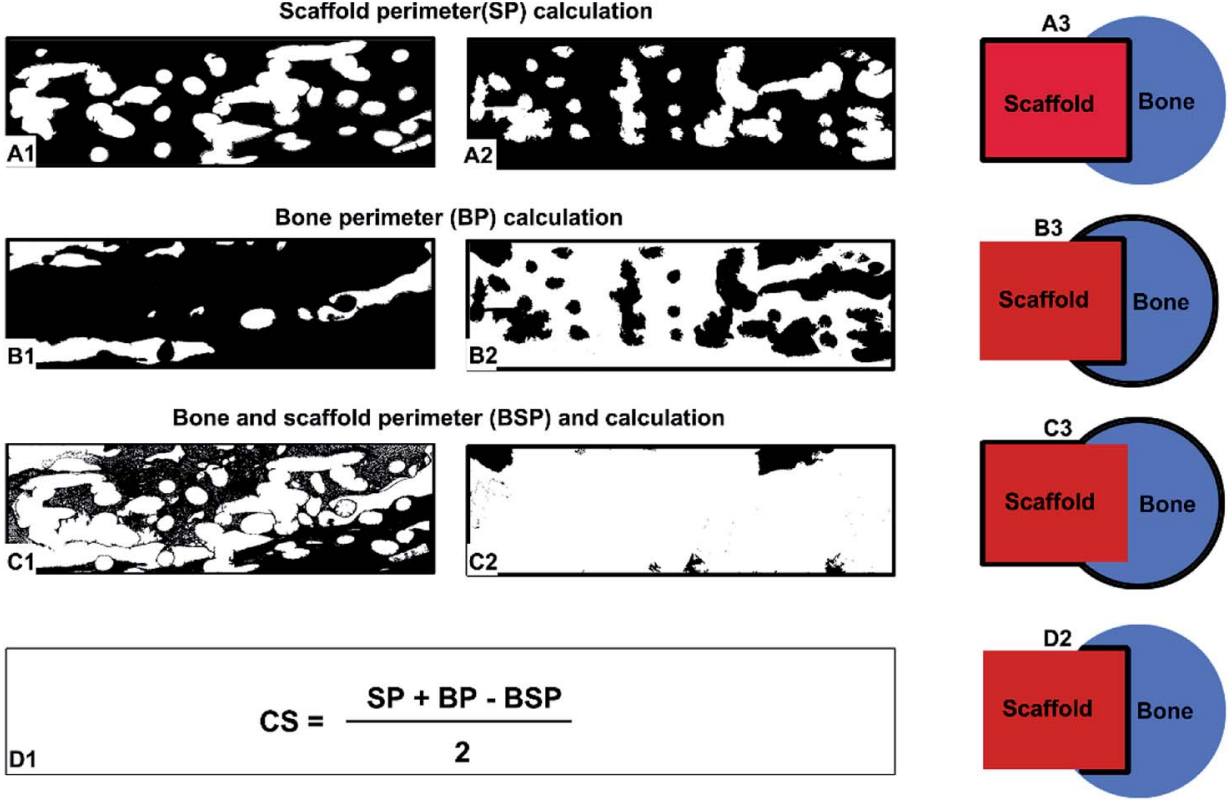

E

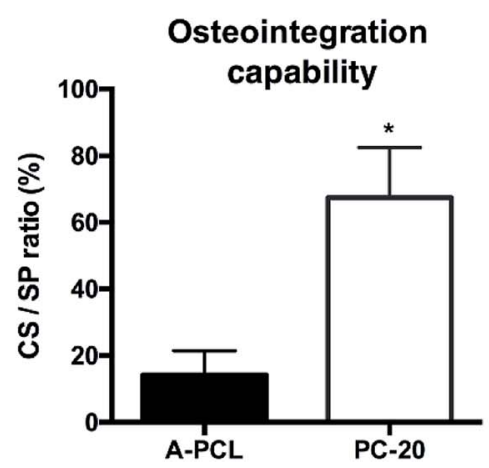

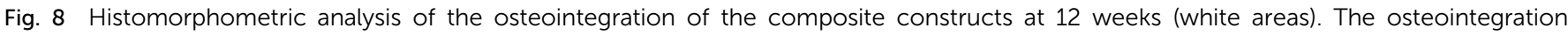

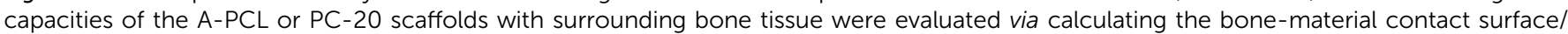

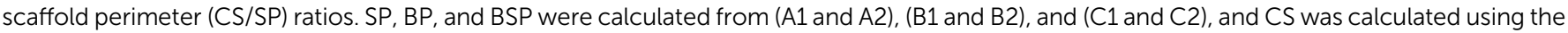

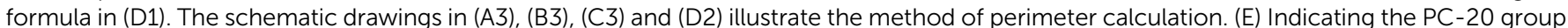

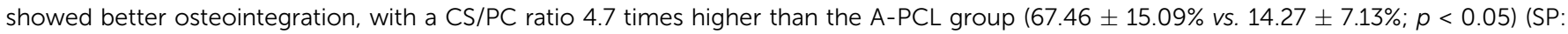
scaffold perimeter; BP: bone perimeter; and BSP: bone and scaffold perimeter; $*: p<0.05)$. 
alkaline treatment because of breaks in the ester chains of the scaffold surface. ${ }^{30}$ Although the EDC cross-linked pure collagen sponge exhibited limited mechanical properties, with a compressive modulus of $0.23 \pm 0.08 \mathrm{MPa}$ (Fig. 3D), this remained less than ideal for surgical handling and bearing osteogenic loads during bone defect healing. ${ }^{10}$ However, the COL-PCL composite constructs display compressive modulus values of $48.61 \pm 4.30,46.48 \pm 5.04$ and $50.89 \pm 6.43 \mathrm{MPa}$, respectively. The results indicate that the compressive modulus of the composites increases synergistically (up to 200-fold), compared with collagen sponge scaffolds alone. The compressive modulus of the composite is close to that of cancellous bone tissue (Fig. 3), and falls within the compressive modulus range for bone tissue regeneration (10-1500 MPa) suggested by Hollister et al. ${ }^{17}$ Further in vivo observations in rabbit radial critical sized bone defects confirmed that the composite scaffold could withstand the temporary physical load-bearing during bone healing. The mechanical strength improvement of the composite constructs can be explained via the classic "brick-and-mortar" reinforcement theory, as stated by Zhou et $a l .{ }^{35}$

Besides the mechanical properties, the biological performance must be meticulously optimized to develop a favourable bone graft substitute for clinical use. Despite their easy processability, the PCL synthetic polymers were generally bio-inert, and could easily elicit in vivo fibrous encapsulation and, ultimately, soft tissue juxtaposition. Such events result in insufficient bone tissue regeneration and compromise the overall success of implants. ${ }^{2,15,36}$ Several strategies have been developed to improve the bioactivity of PCL scaffolds. Previously, Vance et al. demonstrated that alkaline surface treatment could be used to modify the scaffold topography, increasing the surface roughness to decrease fibroblast adhesion and density in vitro. ${ }^{28}$ In our study, the alkaline-activated PCL scaffold (A-PCL) displayed a similar topographical appearance and significantly better hydrophilicity properties and water uptake abilities, but it was still insufficient to improve cell entrapment and proliferation in vitro (Fig. 4B and $\mathrm{C}$ ). Moreover, the in vivo implantation of A-PCL failed at critical sized defect bone regeneration and scaffold-bone osteointegration, eliciting fibrosis encapsulation around the composite scaffolds (Fig. 6 and 7). These results indicate that alkaline treatment alone is not enough to change the passive nature of PCL and achieve successful bone defect regeneration. Therefore, via microporous collagen network incorporation, the composite scaffold achieves favourable bioactivity in vitro, enhancing bone healing and host-scaffold osteointegration capabilities, with less fibrous tissue encapsulation. This is because biomimetic collagen networks served as a bone tissue ECM, enhancing the osteoconductivity and osteoinductivity of the constructs, as previously stated..$^{37}$ The collagen cross-embeds among the voids and coats onto the PCL struts through physical integration and vacuum absorption, increasing the scaffold surface areas and cell anchoring sites for much more efficient cell entrapment and adhesion.9,15 Secondly, the immune-modulating activity of collagen might play a key role in minimize the intensity of fibrous tissue encapsulation, promoting bone tissue regeneration and osteointegration after in vivo implantation. ${ }^{38}$

Although the mechanism of fibrous tissue encapsulation is not clear, it has been demonstrated that a host immune related foreign-body reaction (FBR) plays a critical role. ${ }^{39,40}$ Shortly after biomaterial implantation, nonspecific proteins binding to implanted scaffold surfaces may trigger macrophage recognition and binding. Then macrophages induce fibroblasts to deposit a dense, avascular layer of collagen capsules, which permanently isolates the implant from the organism, resulting in osteointegration failure with native bone tissue. ${ }^{41}$ Secondly, these dense collagenous capsules could block mass transport and/or electric communication between the scaffolds and the body, which could further compromise the rate, quality and efficacy of bone healing and osteointegration. ${ }^{42}$ As a host immune-mediated foreign body response plays a critical role in influencing device functionality and clinical outcome, many researchers have been investigated broad-spectrum antiinflammatories to overcome FBR rejection, including corticosteroids and immunosuppressive agents. ${ }^{31,40,43,44}$ However, many of these drugs are not specific to individual immune populations, having multiple targets and differential effects in vivo. Recently, Wolf et al. indicated that an ECM hydrogel coating could reduce the intensity of the FBR towards a polypropylene scaffold through M2 macrophage polarization, avoiding the side-effects of immunosuppressive agents, and indicating that ECM based methods could be a promising strategy to reduce FBRs and avoid fibrous tissue formation. ${ }^{45}$

Based on results that an ECM could mitigate the FBR and fibrous tissue formation, ${ }^{38}$ in our study a biomimetic collagen ECM-ornamenting strategy was introduced to relieve the FBR and fibrous tissue encapsulation, with the hope of promoting bone regeneration and scaffold osteointegration. The results show better bone regeneration (5.5-fold, Fig. 7A and B), a faster bone deposition rate (1.8-fold, Fig. 7C and D) and osteointegration improvement (4.7-fold) compared to A-PCL scaffolds, indicating that collagen functionalization could enhance the in vivo osteogenesis and osteointegration of 3D PCL scaffolds. This improvement in in vivo osteogenesis is related to enhanced cell attachment and proliferation, as indicated by the in vitro cell experiments. Additionally, the biodegradable collagen networks that homogeneously distribute over and fill the voids among the PCL struts can also serve as a temporary 3D microporous barrier or membrane to prevent fibrous tissue infiltration and preserve the space for new bone formation during the early phase of bone deposition. Moreover, a thin collagen film (approximately $3 \mu \mathrm{m}$ ) coated onto the PCL struts (Fig. 2A), which displayed a synergistic effect with the collagen networks to improve the bioactivity and overcome a host response related FBR to pristine PCL, promoting bone tissue regeneration and osteointegration. Although it is undisputed that the host immune response to implanted materials is indispensable in improving tissue regeneration, ${ }^{43}$ our study demonstrates that a collagen based ECM-ornamenting strategy could foster a pro-healing, rather than a fibrotic, outcome. However, many more studies are needed to fully understand the cellular and biomolecular 
mechanisms of biomaterial induced pro-regenerative, and these should be further elucidated in future investigations.

\section{Conclusions}

To develop an excellent bone graft substitute, balancing mechanical functionality with biological performance for rapid bone tissue ingrowth, in the present study, a collagen functionalization strategy was selected to ornament 3-DP PCL and fabricate highly customized, porous scaffolds with favourable mechanical properties. The composite constructs show outstanding bioactivity and anti-fibrous tissue encapsulation, promoting rapid bone regeneration and osteointegration in a rabbit radius segmental long bone defect model. Moreover, through combining a collagen ornamenting strategy with 3-DP technology, the development of customized, bioactive Col-PCL scaffolds is demonstrated, showing great potential as effective bone graft substitutes for bone tissue regeneration.

\section{Conflicts of interest}

There are no conflicts to declare.

\section{Acknowledgements}

This work was supported by the National Natural Science Foundation of China (81771042, 81772354, 81572137, 81371964); the National Key R\&D Program of China (2016YFC1100100); and the National Young Thousand-Talent Scheme to Zhang Zhi-Yong.

\section{References}

1 B. D. Smith and D. A. Grande, Nat. Rev. Rheumatol., 2015, 11, 213-222.

2 E. M. Bueno and J. Glowacki, Nat. Rev. Rheumatol., 2009, 5, 685-697.

3 Y. Liu, J. Lim and S. H. Teoh, Biotechnol. Adv., 2013, 31, 688705.

4 A. Ho-Shui-Ling, J. Bolander, L. E. Rustom, A. W. Johnson, F. P. Luyten and C. Picart, Biomaterials, 2018, 180, 143-162.

5 J. Wang, D. Wu, Z. Zhang, J. Li, Y. Shen, Z. Wang, Y. Li, Z. Y. Zhang and J. Sun, ACS Appl. Mater. Interfaces, 2015, 7, 26244-26256.

6 A. V. Do, B. Khorsand, S. M. Geary and A. K. Salem, Adv. Healthcare Mater., 2015, 4, 1742-1762.

7 V. Karageorgiou and D. Kaplan, Biomaterials, 2005, 26, 54745491.

8 G. Fernandez de Grado, L. Keller, Y. Idoux-Gillet, Q. Wagner, A. M. Musset, N. Benkirane-Jessel, F. Bornert and D. Offner, J. Tissue Eng., 2018, 9, 2041731418776819.

9 H. Lee and G. Kim, J. Mater. Chem., 2011, 21, 6305.

10 R. J. Kane, H. E. Weiss-Bilka, M. J. Meagher, Y. Liu, J. A. Gargac, G. L. Niebur, D. R. Wagner and R. K. Roeder, Acta Biomater., 2015, 17, 16-25.

11 J. Lim, M. S. K. Chong, J. K. Y. Chan and S.-H. Teoh, Small, 2014, 10, 2495-2502.
12 B. T. Goh, L. Y. Teh, D. B. Tan, Z. Zhang and S. H. Teoh, Clin. Oral Implants Res., 2015, 26, 271-277.

13 I. Zein, D. W. Hutmacher, K. C. Tan and S. H. Teoh, Biomaterials, 2002, 23, 1169-1185.

14 D. W. Hutmacher, T. Schantz, I. Zein, K. W. Ng, S. H. Teoh and K. C. Tan, J. Biomed. Mater. Res., 2001, 55, 203-216.

15 S. Ahn, Y. Kim, H. Lee and G. Kim, J. Mater. Chem., 2012, 22, 15901.

16 F. Pati, T. H. Song, G. Rijal, J. Jang, S. W. Kim and D. W. Cho, Biomaterials, 2015, 37, 230-241.

17 S. J. Hollister, Nat. Mater., 2005, 4, 518-524.

18 S. Lohfeld, S. Cahill, V. Barron, P. McHugh, L. Durselen, L. Kreja, C. Bausewein and A. Ignatius, Acta Biomater., 2012, 8, 3446-3456.

19 S. Buyuksungur, T. Endogan Tanir, A. Buyuksungur, E. I. Bektas, G. Torun Kose, D. Yucel, T. Beyzadeoglu, E. Cetinkaya, C. Yenigun, E. Tonuk, V. Hasirci and N. Hasirci, Biomater. Sci., 2017, 5, 2144-2158.

20 J. Gopinathan, M. M. Pillai, S. Shanthakumari, S. Gnanapoongothai, B. K. Dinakar Rai, K. Santosh Sahanand, R. Selvakumar and A. Bhattacharyya, Nanomedicine, 2018, 14, 2247-2258.

21 M. T. Arafat, C. X. Lam, A. K. Ekaputra, S. Y. Wong, X. Li and I. Gibson, Acta Biomater., 2011, 7, 809-820.

22 A. M. Ferreira, P. Gentile, V. Chiono and G. Ciardelli, Acta Biomater., 2012, 8, 3191-3200.

23 A. Oyane, M. Uchida, C. Choong, J. Triffitt, J. Jones and A. Ito, Biomaterials, 2005, 26, 2407-2413.

24 Z. Xia, X. Yu, X. Jiang, H. D. Brody, D. W. Rowe and M. Wei, Acta Biomater., 2013, 9, 7308-7319.

25 J. P. Schmitz and J. O. Hollinger, Clin. Orthop. Relat. Res., 1986, 299-308.

26 W. Zhang, G. Wang, Y. Liu, X. Zhao, D. Zou, C. Zhu, Y. Jin, Q. Huang, J. Sun, X. Liu, X. Jiang and H. Zreiqat, Biomaterials, 2013, 34, 3184-3195.

27 Y. Li, Z. G. Wu, X. K. Li, Z. Guo, S. H. Wu, Y. Q. Zhang, L. Shi, S. H. Teoh, Y. C. Liu and Z. Y. Zhang, Biomaterials, 2014, 35, 5647-5659.

28 R. J. Vance, D. C. Miller, A. Thapa, K. M. Haberstroh and T. J. Webster, Biomaterials, 2004, 25, 2095-2103.

29 H. A. Declercq, T. Desmet, P. Dubruel and M. J. Cornelissen, Tissue Eng., Part A, 2014, 20, 434-444.

30 A. Oyane, M. Uchida, Y. Yokoyama, C. Choong, J. Triffitt and A. Ito, J. Biomed. Mater. Res., Part A, 2005, 75, 138-145.

31 J. C. Doloff, O. Veiseh, A. J. Vegas, H. H. Tam, S. Farah, M. Ma, J. Li, A. Bader, A. Chiu, A. Sadraei, S. ArestaDasilva, M. Griffin, S. Jhunjhunwala, M. Webber, S. Siebert, K. Tang, M. Chen, E. Langan, N. Dholokia, R. Thakrar, M. Qi, J. Oberholzer, D. L. Greiner, R. Langer and D. G. Anderson, Nat. Mater., 2017, 16, 671-680.

32 J. A. Inzana, D. Olvera, S. M. Fuller, J. P. Kelly, O. A. Graeve, E. M. Schwarz, S. L. Kates and H. A. Awad, Biomaterials, 2014, 35, 4026-4034.

33 A. Goyanes, U. Det-Amornrat, J. Wang, A. W. Basit and S. Gaisford, J. Controlled Release, 2016, 234, 41-48.

34 S. M. Giannitelli, D. Accoto, M. Trombetta and A. Rainer, Acta Biomater., 2014, 10, 580-594. 
35 C. Zhou, X. Ye, Y. Fan, L. Ma, Y. Tan, F. Qing and X. Zhang, Biofabrication, 2014, 6, 035013.

36 M. Pilia, T. Guda and M. Appleford, BioMed Res. Int., 2013, 2013, 458253.

37 Z. Xia, X. Yu and M. Wei, J. Biomed. Mater. Res., Part B, 2012, 100, 871-881.

38 C. Chu, J. Deng, X. Sun, Y. Qu and Y. Man, Tissue Eng., Part B, 2017, 23, 421-435.

39 W. Kenneth Ward, J. Diabetes Sci. Technol., 2008, 2, 768-777.

40 N. M. Vacanti, H. Cheng, P. S. Hill, J. D. T. Guerreiro, T. T. Dang, M. Ma, S. Watson, N. S. Hwang, R. Langer and D. G. Anderson, Biomacromolecules, 2012, 13, 3031-3038.

41 L. Zhang, Z. Cao, T. Bai, L. Carr, J.-R. Ella-Menye, C. Irvin, B. D. Ratner and S. Jiang, Nat. Biotechnol., 2013, 31, 553-556.
42 X. Zhang, C. Zhang, Y. Lin, P. Hu, Y. Shen, K. Wang, S. Meng, Y. Chai, X. Dai, X. Liu, Y. Liu, X. Mo, C. Cao, S. Li, X. Deng and L. Chen, ACS Nano, 2016, 10, 7279-7286.

43 K. Sadtler, K. Estrellas, B. W. Allen, M. T. Wolf, H. Fan, A. J. Tam, C. H. Patel, B. S. Luber, H. Wang, K. R. Wagner, J. D. Powell, F. Housseau, D. M. Pardoll and J. H. Elisseeff, Science, 2016, 352, 366-370.

44 K. Qiu, B. Chen, W. Nie, X. Zhou, W. Feng, W. Wang, L. Chen, X. Mo, Y. Wei and C. He, ACS Appl. Mater. Interfaces, 2016, 8, 4137-4148.

45 M. T. Wolf, C. L. Dearth, C. A. Ranallo, S. T. LoPresti, L. E. Carey, K. A. Daly, B. N. Brown and S. F. Badylak, Biomaterials, 2014, 35, 6838-6849. 